\title{
3 Research Sourere \\ Health Related Quality of Life in Men After Total Hip Replacement
}

\section{Monika Białkowska}

District Hospital of Orthopaedics and Trauma Surgery

\section{Tomasz Stołtny}

District Hospital of Orthopaedcis and Trauma Surgery

Jarosław Pasek ( $\square$ jarus_tomus@o2.pl )

Faculty of Health Sciences Jan Długosz University in Częstochowa https://orcid.org/0000-0001-6181-337X

Michał Mielnik

District Hospital of Orthopaedics and Trauma Surgery

\section{Karol Szyluk}

District Hospital of Orthopaedics and Trauma Surgery

Sławomir Kasperczyk

Departrment of Biochemistry Medical University of Silesia

Grzegorz Cieślar

Department of Internal Medicine Medical University of Silesia

\section{Bogdan Koczy}

District Hospital of Orthopaedics Trauma and Surgery

\section{Research article}

Keywords: degenerative hip disease, surgical treatment, cementlesstotal hip arthroplasty, Quality of Life

Posted Date: June 8th, 2020

DOI: https://doi.org/10.21203/rs.3.rs-33346/v1

License: (a) This work is licensed under a Creative Commons Attribution 4.0 International License. Read Full License 


\section{Abstract}

Background: Hip osteoarthritis is a substantial health-related, social, and economic problem. The aim of the study was assessment of life quality in men with degenerative hip disease, after total hip arthroplastyor metaphyseal surgery.

Material and methods: 118 male patients were subjects of the study, being in the age range from 31 to $79 y e a r s$ of age, qualified for the procedure of total hip arthroplasty. The study was a questionnaire study, in which for assessing Quality of Life in patients, the abbreviated version of WHOQoL-BREF,as well as EQ-5D-5L, and SF-36 scale. The assessment of life quality was performed before the surgery, during the first follow-up visit (6 weeks after surgery) and 6 months after the surgery.

Results: The results from WHOQoL-BREF questionnaire demonstrated a statistically significant improvement regarding the assessed quality of life after the surgery -in the 6th week after the procedure by $4 \%$ and $21 \%$ $(p<0.001)$, after 6 months by $13 \%$ and $42 \%(p<0.001)$, respectively. The most significant improvement was found in the somatic domain - by $5 \%$ after 6 weeks, and by $6 \%$ after 6 months( $(p<0.001)$. The quality of life according to the EQ-5D-5L questionnaire revealed statistically significant improvement after 6 weeks, by 18 $24 \%(p<0.001)$, while after 6 months by $41-48 \%(p<0.001)$. The most considerable improvement was achieved in reducing pain complaints and enhancing the ability to move.In the SF-36 questionnaire, in turn, statistically significant improvement 6 months after the surgery refer to both physical (by $44 \%$ ), and mental condition(by $54 \%)(p<0.001)$.

Conclusions: The total hip arthroplasty results in improvement quality of life in examined group ofmen withhip osteoarthrosis, as early as 6 weeks after the procedure, while the improvement is more perceptible 6 months after the procedure, mainly the less severe pain experienced, and better functioning of examined patients.

\section{Background}

In recent years the assessment of Quality of Life (QoL) has been interest to many medical disciplines. In those studies, the emphasis is on the present overall health status, alternatively the assessment concerns the period following a disease the person suffered from. Health status analysis may be treated in objective way, in such a case the economic conditions, spare time, social security, housing conditions, natural environment, health, social environment are considered; or in subjective way, in which case assessment is made concerning individual perception of happiness, anxieties, hope, loneliness, well-being, and satisfaction. The perception quality of life depends also on the present health condition, as diseases may have a decisive influence upon quality of life perception, on several levels simultaneously [1, 2].

The development of research concerning quality of life connected with changes in health condition allowed to isolate the notion quality of life dependent on health condition, disease processes, in reference to natural aging process. Regardless the definition assumed, research concerning quality of life should take into account such factors as: man's functional capacity, the way a patient perceives her/his life situation, level of satisfaction with life, well-being, as well as disease manifestations and physical condition resulting from disease processes and age. In order to correctly determine QoL, also psychosocial resources should be considered, as well as risk factors in the period preceding the disease (age, sex, education level socio- 
economic status, family status), also social bonds and support, interests, and level of medical expertise [3, 4]. At present, life quality assessment used by many authors takes into account not only the clinical assessment but also functional condition of subjects $[4,5]$.

Degenerative hip disease is among the most common diseases affecting locomotor system, posing a serious health, social, and economic threat. Hip degeneration, called coxarthrosis, affects some $4 \%$ of the population, according to statistics. It affects mainly people over 60 years of age, although it may also occur in younger persons, under 40 years of age $[6,7]$.

Degenerative hip disease is a progressive disease. It develops slowly, while intense pain and limited mobility in joint leads to disability and reduced quality of life. In the circumstances where pain occurs at rest and at night, and mobility is severely reduced in the hip, the possibility of performing arthroplasty should be considered. Surgical treatment is a treatment of choice, recommended in patients in advanced stages of the disease, for whom symptomatic treatment failed to give satisfactory results [8, 9].

\section{Aim Of The Study}

The aim of the study was assessment quality of life in men with degenerative hip disease, after total hip arthroplasty or metaphyseal surgery.

\section{Material And Methods}

The study was conducted on male patients, qualified for total or cementless metaphyseal hip arthroplasty procedure, in the District Hospital of Orthopaedics and Trauma Surgery in xxx in 2018-2019 years. On admission to the ward, patient's history was taken and physical examination was performed. The surgical intervention was performed under subarachnoid anaesthesia or general endotracheal anaesthesia.

In total, 118 patients were examined, their age range being between 31 and 79 years (mean age 58 years). Inclusion criteria for the study comprised: male sex (due to the specification of orthopaedic department and fact, that in this ward only men are treated), primary degenerative changes in the hip joint in the age range of 30-80 years, and patient's consent for taking part in the questionnaire study. Exclusion criteria were as follows: female sex, femoral neck fracture, as well as absence of patient's consent for taking part in the questionnaire study.

In the study presented here, the authors used the available Quality of Life (QoL) questionnaires and assessed that quality over a shorter follow-up period, before the surgery, 6 weeks after surgery and in medium-term (6 months after hip arthroplasty). The questionnaire was anonymous, and participation in the study was voluntary. The abbreviated version of the WHOQoL-BREF (World Health Organization / Quality of Life - BREF) was used, consisting of 26 questions concerning overall quality of life, self-assessment of health condition and other spheres of life four weeks before; the EuroQol - EQ-5D-5L questionnaire, assessing the health condition on the day of examination, in which the questions referred to mobility, self-service abilities, performing everyday activities such as: work, education, household chores, experiencing pain/discomfort, as well as anxiety / depression; and the SF-36 questionnaire, assessing 8 dimensions of health: physical 
functioning, social functioning, role limitation regarding physical problems, role limitation regarding emotional problems, mental health, vitality, and general health assessment [10-12].

\section{Statistical analysis}

For the purpose of statistical analysis, Statistica 12 PL and MS Excel 2016 have been used. Measurable values have been presented as arithmetic mean with standard deviation (SD). Chi-squared test was used for assessing quality variables. The Shapiro-Wilk test was used to assess the distribution normality, while Levene's test was used to check the equality of variances. For the sake of comparing two groups, T-test, T-test with independent variance estimate or U Mann-Whitney U test was used. Dependent variables were analysed by T-test of dependent variables or Wilcoxon test. As threshold for statistical significance, the level of $p<0.05$ was assumed for changes observed.

\section{Results}

Clinical characteristics of the studied patient population are presented in Table 1. In the studied population, the mean age was $58 \pm 11$ years, BMI values amounted to $29.2 \pm 5.24 \mathrm{~kg} / \mathrm{m}^{2}$, while the average duration of degenerative disease was $6.0 \pm 5.21$ years.

Table 1

Clinical characteristics of the studied population of patients.

\begin{tabular}{|c|c|c|}
\hline & $\begin{array}{l}\text { Mean } \\
\text { /percent }\end{array}$ & SD \\
\hline age [in years] & 58 & 11 \\
\hline work experience [in years] & 34.3 & 10.4 \\
\hline body mass [kg] & 89.8 & 14.5 \\
\hline height [cm] & 174.7 & 7.47 \\
\hline BMI index value $\left[\mathrm{kg} / \mathrm{m}^{2}\right]$ & 29.2 & 5.24 \\
\hline duration of degenerative disease [in years] & 6.0 & 5.21 \\
\hline percentage of town dwellers [\%] & 66.7 & \\
\hline percentage of blue collar workers [\%] & 85 & \\
\hline cardiovascular system diseases [\%] & 97 & \\
\hline diabetes [\%] & 19.7 & \\
\hline percentage of patients operated on the right side [\%] & 52 & \\
\hline
\end{tabular}

Based on the analysis of WHOQoL-BREF questionnaire results, it has been stated that quality of life and satisfaction with health status (values of both factors in the 1 to 5 scale) improved, with statistical 
significance, 6 weeks after intervention by $4 \%$ and $21 \%$, and 6 months after the procedure by $13 \%$ and $42 \%$, respectively (Fig. 1).

In subsequent questions from the WHOQoL-BREF questionnaire, improvement in the quality of life was noted for specific areas. The most substantial, and statistically significant improvement was observed in the somatic domain - by $5 \% 6$ weeks after surgery, and by $6 \% 6$ months after it. As regards other domains: psychological, social and environmental ones, a statistically significant improvement was also noticed - by $4 \% 6$ weeks after surgery, and by 7-9\% 6 months after it (Fig. 2).

Based on the analysis of the quality of life results obtained from the questionnaire concerning health (EQ-5D$5 \mathrm{~L})$ the following have been improved, with statistical significance: mobility, self-service, daily activity, pain / discomfort, and anxiety or depression - by 18-24\% $(p<0.001) 6$ weeks after surgical intervention, and by $41-$ $48 \%$ ( $p$ < 0.001) 6 months after hip atrhroplasty. The most significant improvement concerned pain and mobility (Fig. 3).

Moreover, in subjective assessment of present health condition (scale 0 to 100) that condition was statistically significantly improved - by $20 \% 6$ weeks after surgery, and by $38 \% 6$ months after hip arthroplasty (Table 2 and Fig. 4).

Table 2

Subjective assessment of health condition for patients before, 6 weeks after surgery, and 6 months after total hip arthroplasty.

\begin{tabular}{|c|c|c|c|c|c|c|c|c|c|c|}
\hline \multirow[t]{2}{*}{$n=118$} & mean & SD & mean & SD & \multirow{2}{*}{$\begin{array}{l}\% \\
\text { of } \\
\text { change }\end{array}$} & \multirow{2}{*}{$\begin{array}{l}\mathrm{P} \\
\text { value }\end{array}$} & mean & SD & \multirow{2}{*}{$\begin{array}{l}\% \\
\text { of } \\
\text { change }\end{array}$} & \multirow{2}{*}{$\begin{array}{l}\mathrm{P} \\
\text { value }\end{array}$} \\
\hline & \multicolumn{2}{|c|}{ before surgery } & \multicolumn{2}{|c|}{$\begin{array}{l}6 \text { weeks after } \\
\text { hip } \\
\text { arthroplasty }\end{array}$} & & & \multicolumn{2}{|c|}{$\begin{array}{l}6 \text { months after } \\
\text { hip } \\
\text { arthroplasty }\end{array}$} & & \\
\hline EQ-1 & 3.46 & 0.74 & 2.68 & 0.57 & $-23 \%$ & $\stackrel{<}{0.001}^{<}$ & 1.97 & 0.49 & $-43 \%$ & $\begin{array}{l}< \\
0.001\end{array}$ \\
\hline EQ-2 & 2.88 & 1.01 & 2.36 & 0.72 & $-18 \%$ & $\grave{0}_{0.001}$ & 1.67 & 0.57 & $-42 \%$ & $\begin{array}{l}< \\
0.001\end{array}$ \\
\hline EQ-3 & 3.09 & 0.95 & 2.49 & 0.70 & $-19 \%$ & $\begin{array}{l}< \\
0.001\end{array}$ & 1.81 & 0.52 & $-41 \%$ & $\begin{array}{l}< \\
0.001\end{array}$ \\
\hline EQ-4 & 3.43 & 0.85 & 2.61 & 0.68 & $-24 \%$ & $\stackrel{<}{0.001}^{<}$ & 1.90 & 0.50 & $-45 \%$ & $\begin{array}{l}< \\
0.001\end{array}$ \\
\hline EQ-5 & 2.78 & 1.17 & 2.14 & 0.85 & $-23 \%$ & $\stackrel{<}{0.001}^{<}$ & 1.46 & 0.61 & $-48 \%$ & $\begin{array}{l}< \\
0.001\end{array}$ \\
\hline $\begin{array}{l}\text { Present } \\
\text { health } \\
\text { condition, } \\
\text { scale 0 } \\
\text { to100 }\end{array}$ & $52.1 \%$ & $18.5 \%$ & $62.5 \%$ & $14.1 \%$ & $20 \%$ & $\begin{array}{l}< \\
0.001\end{array}$ & $72.2 \%$ & $11.9 \%$ & $38 \%$ & $\begin{array}{l}< \\
0.001\end{array}$ \\
\hline
\end{tabular}

The analysis of results according to SF-36 questionnaire for assessing the quality of life revealed a statistically significant improvement regarding physical condition, daily activity, and functional limitations - by $14 \%(p<0.001) 6$ weeks after surgery, and by $44 \%(p<0.001) 6$ months after hip arthroplasty. Interpretation of 
results concerning mental condition, comprising overall health perception, vitality, emotional functioning, and mental health showed improvement in comparison with the initial values 6 weeks after surgery by $21 \%(p<$ $0.001)$, and 6 months after hip arthroplasty by $54 \%(p<0.001)$. Moreover, in the subjective assessment the Quality of Life Index (maximum amount of points in the scale - 171) improved with statistical significance by $19 \% 6$ weeks after surgery $(p<0.001)$, and by $51 \% 6$ months after hip arthroplasty $(p<0.001)$ (Fig. 5).

\section{Discussion}

Increased interest in studies concerning quality of life results from the necessity of improving the efficacy of health care and objectivizing treatment results. The complex nature of problems encompassing the wide range of physical and mental health, as well as the environment in which patients function, indicates the legitimacy and valuability of applying various methods and tools for measuring health condition, useful in planning and providing complex care given by various professionals, in preparing patients for self-care $[2,13]$. Interest in quality of life in contemporary medicine is also a form of response to challenges and demands it faces, which result from increasing occurrence of chronic and progressing diseases in societies. It is also related to increasing life expectancy, thus the period in which patients's functioning is substantially disturbed is extended, which influences the patient's life situation and its subjective reception $[5,14]$.

Using a specific questionnaire in assessing quality of life depends on the aim of research the author wants to carry out, as it has to be suitable for the clinical situation under consideration [11]. As demonstrated in the study of Jin X et al., who compared the validity of using questionnaires EQ-5D-5L and EQ-5D-3L serving the purpose of assessing the quality of life, the EQ-5D-5L questionnaire proved to be a more sensitive research tool in case of patients after total hip reconstruction (THR), as well as total knee reconstruction (TKR). Thus, the use of EQ-5D-5L questionnaire in the above study seems fully justified [15].

The measurements of quality of life during clinical examination allowed to confirm beneficial results of treatment. As demonstrated in research, the quality of life assessed acc. to WHOQol-BREF questionnaire, concerning overall quality of life, self-assssment, and health condition, improved in the study group of patients as early as 6 weeks after surgery $(p<0.001)$. That result was also beneficial as showed by the subsequent checking, carried out 6 months after hip arthroplasty $(p<0.001)$.

Snell DL et al. evaluated the quality of life in patients after total hip arthroplasty (THA) and total knee arthroplasty (TKA), using also the WHOQoL-BREF questionnaire, 6, 12, 24, and 60 months after the intervention. The results of their study demonstrated a positive correlation between the clinical condition and the assessed psychological aspects which influence the quality of life of treated patients [16].

Also Lapaj L. et al. evaluated the quality of life of 62 patients ( 44 female and 18 male ones) before and after hip arthroplasty procedure (mean follow-up: 12-18 months) using modified WHOQoL-100 questionnaire. The results of their studies confirmed significant beneficial influence of the surgical intervention upon the physical and psychological level of independence, as well as overall qualify of life in patients who were subjects of surgical treatment, whereas no influence of the surgical procedure upon the level of social independence was demonstrated [17]. 
It is worthwhile to refer here to the results of the study by Peeters CM et al. who made a metaanalysis of 49 studies from available databases, which refer to the assessment of health status (HR) and health-related quality of life (HRQoL) in patients over 65 years of age, after arthoplasty or capoplasty due to femur fracture. The analysis comprised the period of 6 months after surgical intervention, that is the mean follow-up. The research demonstrated that quality of life was significantly higher in the patients who underwent arthroplasty, in comparison with patients on whom capoplasty was performed [18].

In the study reported here, the authors also used most widely generic EQ-5D-5L questionnaire for evaluation of qualify of life, which also revealed a significant improvement in quality of life as early as 6 weeks after the surgery $(p<0.001)$, while the degree of improvement achieved $(41-48 \%)$ was significantly higher 6 months after hip arthroplasty. The most remarkable improvement was noted in the reduction of pain, as well as enhanced mobility $(p<0.001)$.

Similar results were achieved by Costa ML. et al., who compared the clinical condition assessment using Oxford Hip Score (OHS), and quality of life using the EuroQol - EQ-5D-5L in patients after total hip arthroplasty, in comparison with resurfacing arthroplasty, in the group of 122 patients over 18 years of age, follow-up 5 years [19].

Miao N.F. et al. made the assessment of clinical condition (Oxford Hip Score and Oxford Knee Score) as well as quality of life (EQ-5D-5L) in patients who had undergone total hip arthroplasty and knee arthroplasty. The assement was made before surgical intervention, 6 weeks after it, as well as 3 and 6 months after. The authors demonstrated that functional condition and quality of life improved, while the results obtained were significantly higher in the female group after knee arthroplasty $(p<0.05)$ [20].

In another study, Roidis N.T. et al. assessed the quality of life using EQ-5D-5L questionnaire in 82 women after total hip arthroplasty in long follow-up (1, 2, and 12 years after surgical intervention). The results of that study demonstrated statistically significant improvement in joint mobility, as well as reduction of pain experienced, in all the three periods of observation assessed [21].

Another questionnaire used in the study reported here is SF-36 questionnaire. It assessed the physical and mental condition of the study subjects. Also in this case, quality of life improved, with statistical significance, both 6 weeks after surgery (by 21\%) ( $<<0.001)$, and 6 months after hip arthroplasty (by 54\%) $(p<0.001)$.

SF-36 questionnaire was also used by Bahardoust M. et al., who assessed the quality of life (HRQoL), clinical condition, and socio-demographic aspects in 217 Iranian patients after total hip replacement. The mean follow-up was 18-27 weeks after the surgical intervention. The results demonstrated that hip replacement procedure influenced the improvement in the quality of life assessed, which directly influenced the clinical condition of patients, while the improvement achieved was not a constant value, and changed over the time range assessed [22].

Neuprez A. et al., in turn, assessed the influence of total hip arthroplasty (THA) and total knee arthroplasty (TKA) upon the pain experienced and quality of life in late follow-up (5 years after the intervention) in the group of 626 surgical patients. Validated specific Western Ontario and McMaster Universities Arthritis Index (WOMAC) and generic (SF-36 and EQ-5d-5L) instruments assessing quality of life were used prior to surgery 
and yearly, thereafter. The beneficial effect on quality of life observed during the first year after hip and knee arthroplasty (THA and TKA) was maintained for up to 5 years. More than $3 / 4$ of the patients in this study experienced a good outcome (86.04\% in THA group and $79.91 \%$ in TKA group) [23].

Hofstede S.N. et al. conducted a study concerning the assessment of pre-surgery determinants upon the perception of quality of life, functioning, and pain experienced, in patients from 20 cohorts with OA $(n=1783)$ TKA and $n=2400$ (THA) in the Netherlands. The authors examined the influence of age, gender, BMI, and preoperative values of HRQoL, functioning, and pain on postoperative status and total improvement. For each increase in preoperative point in HRQoL, the postoperative HRQoL increased by 0.51 points in TKA and by 0.37 points in THA in SF-36 scale. Patients with a better preoperative health status have better outcomes, but less improvement. Even though the independent effects may seem small, combined results of preoperative variables may result in larger effects on postoperative outcomes [24].

Balik M.S. et al., have also assessed, like the authors of the a/m study, the quality of life in the group of 150 patients after primary hip arthroplasty. The authors additionally assessed the psychiatric symptoms before and after the operation, in order to demonstrate disease-related socio-demographical characteristics of the patient. The Quality of Life Scale Short Form (SF-36), Beck Depression Inventory (BDI), Beck Anxiety Inventory (BAI), Harris Hip Score (HHS) and Visual Analog Scale (VAS) were implemented in the preoperative period and at 6th and 12th week after the operation. After total hip arthroplasty, significant improvement was observed in quality of life (SF-36), depression, and pain scores (VAS) [25].

It is generally believed that diseases disturb the functioning of men to various degrees, affecting man's quality of life in its many dimensions. That is why in the process of treatment, besides achieving medical targets, an important role belongs to improving the comfort of living for the treated patient. Total hip arthroplasty is the method of choice in the treatment of advanced stages of degenerative changes in hip joint. The aim of the surgical procedure is to increase hip mobility, to enable the patient to return to professional and social activity. The above-mentioned factors have significant influence upon the improvement of patient's quality of life.

\section{Conclusions}

1. Total hip arthroplasty procedure results in statistically significant improvement quality of life in examined group of men with degenerative changes, as early as 6 weeks after the surgery, while the degree of improvement increases further 6 months after hip arthroplasty.

2. The improvement achieved concerned both physical and mental health, mainly the less severe pain experienced, and better functioning of examined patients.

\section{Abbreviations}

QoL - Quality of Life

HRQoL - Health-RelatedQuality of Life

WHOQoL-BREF - World Health Organization / Quality of Life - BREF) 
EQ-5D-5L - EuroQol questionnaire

SF-36 - Scale Short Form 36 questionnaire

BMI - Body Mass Index

THR - Total hip reconstruction

TKR - Total knee reconstruction

THA - Total hip arthroplasty

TKA - Total knee arthroplasty

OHS - Oxford Hip Score

WOMAC - Western Ontario and McMaster Universities Arthritis Index

BDI - Beck Depression Inventory

BAI - Beck Anxiety Inventory

HHS - Harris Hip Score

VAS - Visual AnalogScale

\section{Declarations}

\section{Ethicsapproval and consent to participate}

Based on the meeting of the Bioethics Committees of the Silesian Medical Chamber on 30 July 2018, the above-mentioned surveys do not require the consent of the Bioethics Committees of the Silesian Medical Chamber. (ŚIL/KB/1044p/18).All qualified patients signed a written consent for participation in this study.

\section{Consent for publication}

Not applicable.

\section{Availability of data and materials}

The datasetsused and/oranalysedduring the currentstudyareavailable from the correspondingauthor on reasonablerequest.

\section{Competinginterests}

The author's declare that there is no conflict of interests regarding the publication of this paper.

\section{Funding}


Not applicable.

\section{Authors' contributions}

MB - study design, data collection, data interpretation, manuscript preparation

TS - study design, data collection, data interpretation, manuscript preparation

JP - study design, data collection, data interpretation, manuscript preparation, literature search

MM - data collection, data interpretation, manuscript preparation

$\mathrm{KSz}$ - data collection, manuscript preparation, literature search

SK - data collection, literature search, statistical analysis

GC - study design, data interpretation, manuscript preparation

BK - study design, data interpretation

\section{Acknowledgements}

Not applicable.

\section{References}

1. Uysal M. Sirgy J. Quality-of-life indicators as performance measures. Annals of Tourism Research. 2019;76:291-300.

2. Zięba M, Cisoń-Apanasewicz U. Quality of Life in medicine. Forum Reumatol. 2017;3(1):5-12.

3. Schalock RL. The concept of quality of life: what we know and do not know. J Intellect Disabil Res. 2004;48(3):203-16.

4. Alonso J, Ferrer M, Gandek B, et al IQOLA Project Group. Health-related quality of life associated with chronic conditions in eight countries: results from the International Quality of Life Assessment (IQOLA) Project. Qual Life Res. 2004;13(2):283-98.

5. Pasek J, Opara J, Pasek T, et al. The meaning of Quality of Life in rehabilitation. Physiotherapy. 2007;15(3):3-8.

6. Wiecheć M. Why important in cooperation physiotherapist, orthopaedics and personal coach - on ostearthritis joints example. Practical Physiother Rehabil. 2019;108:36-8.

7. Azam MQ, Sadat-Ali M. Quality of Life in sickle cell patients after cementless total hip arthroplasty. J Arthroplasty. 2016;31(11):2536-41.

8. Tomaszewski W, Kotela I, Kawik $Ł$, et al. Quality of Live of patients in the evaluation of outcomes of short stem hip arthroplasty for hip osteoarthritis. Ortop Traumatol Rehabil. 2003;5(6):439-57.

9. Shan L, Shan B, Graham D, Saxena A. Total hip replacement: a systematic review and metaanalysis on mid-term quality of life. Osteoarthritis Cartilage. 2014;22(3):389-406. 
10. Jaracz K, Kalfoss M, Górna K, et al. Quality of life in Polish respondents: psychometric properties of the Polish WHOQoL-Bref. Scand J Caring Sci. 2006;20(3):251-60.

11. Cieślik B, Podbielska H. Overview of chosen questionnaire concerning Quality of Life. Acta Bio-Opt et Inform Med. 2015;21:102-35.

12. Tylka J, Piotrowicz R. Quality of life SF-36 questionnaire - the Polish version. Kardiol Pol. 2009;67:116669.

13. Sierakowska M. Quality of Life in medicine. Forum Reumatol. 2017;3(16):5-12.

14. Alexiou KI, Roushias A, Varitimidis SE, Malizos KN. Quality of life and psychological consequences in elderly patients after a hip fracture: a review. Clin Interv Aging. 2018;24;143 - 50.

15. Jin X, Al Sayah F, Ohinmaa A, et al. Responsiveness of the EQ-5D-3L and EQ-5D-5L in patients following total hip or knee replacement. Qual Life Res. 2019;28(9):2409-17.

16. Snell DL, Siegert RJ, Surgenor LJ, et al. Evaluating quality of life outcomes following joint replacement: psychometric evaluation of a short form of the WHOQoL-Bref. Qual Life Res. 2016;25(1):51-61.

17. Lapaj L, Kokoszka P, Jurga M, et al. Quality of life in patients before and after total hip arthroplasty. Chir Narz Ruchu Ortop Pol. 2007;72(4):269-74.

18. Peeters CM, Visser E, Van de Ree CL, et al. Quality of life after hip fracture in the elderly: A systematic literature review. Injury. 2016;47(7):1369-82.

19. Costa ML, Achten J, Foguet P, Parsons NR. Comparison of hip function and quality of life of total hip arthroplasty and resurfacing arthroplasty in the treatment of young patients with arthritis of the hip joint at 5 years. BMJ Open. 2018;8(3):849-55.

20. Miao NF, Lin RN. A prospective study of joint function and the quality of life of patients undergoing total joint replacement. Int J Nursing Pract. 2018;24(5):76-83.

21. Roidis NT, Pollalis AP, Hartofilakidis GC. Total hip arthroplasty in young females with congenital dislocation of the hip, radically improves their long-term quality of life. J Arthroplasty. 2013;28(7):120611.

22. Bahardoust M, Hajializade M, Amiri R, et al. Evaluation of health-related quality of life after total hip arthroplasty: a case-control study in the Iranian population. BMC Musculoskelet Disord. 2019;20(1):1-7.

23. Neuprez A, Neuprez AH, Kaux JF, et al. Total joint replacement improves pain, functional quality of life, and health utilities in patients with late-stage knee and hip osteoarthritis for up to 5 years. Clin Rheumatol. 2019;13:67-72.

24. Hofstede SN, Gademan MGJ, Stijnen T, et al. The influence of preoperative determinants on quality of life, functioning and pain after total knee and hip replacement: a pooled analysis of Dutch cohorts. BMC Musculoskelet Disord. 2018;19(1):68-74.

25. Balik MS, Hocaoğlu Ç, Erkut A, et al. Evaluation of the Quality of Life and psychiatric symptoms of patients with primary coxarthrosis after total hip arthroplasty. Acta Chir Orthop Traumatol Cech. 2017;84(6):436-40.

\section{Figures}






\section{Figure 1}

Assessment of the quality of life and satisfaction with health status before, 6 weeks and 6 months after total hip arthroplasty, according to WHOQoL-BREF questionnaire. ${ }^{*} p<0.05$, ${ }^{*} \mathrm{p}<0.01,{ }^{\star \star *} \mathrm{p}<0.001$, comparedto presurgery values, $++p<0.01,+++p<0.001$ comparedto values after surgery ( 6 weeks).

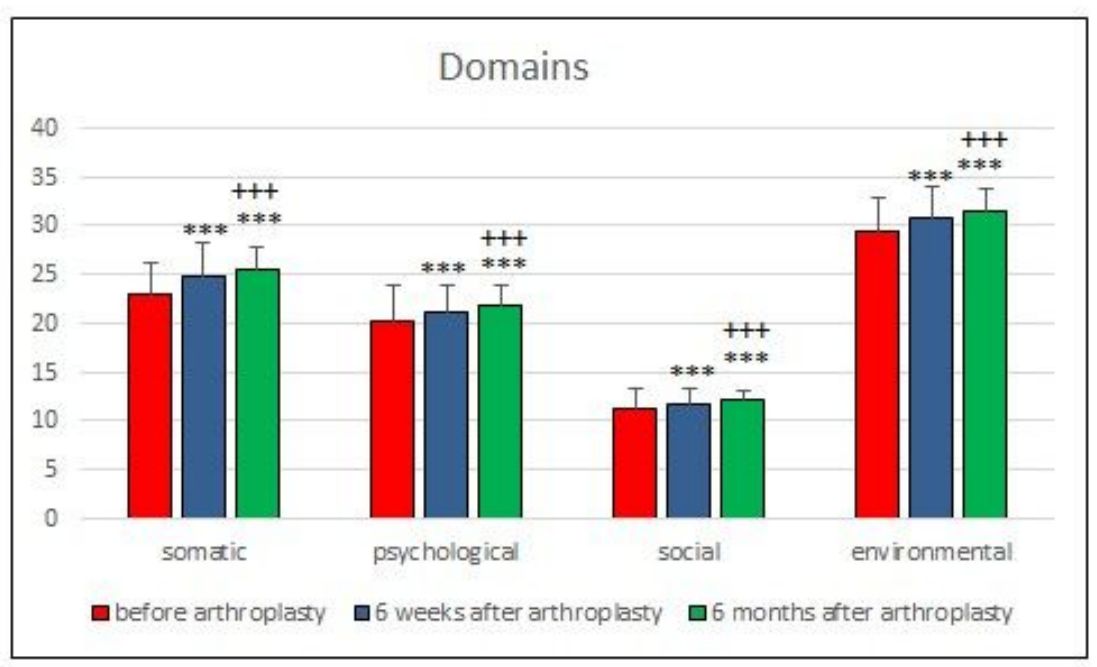

\section{Figure 2}

Assessment of quality of life according to WHOQoL-BREF questionnaire before, 6 weeks and 6 months after total hip arthroplasty. ${ }^{* *} p<0.001$ compared to the values before the procedure, $+++p<0.001$ comparedto the values after surgery (6 weeks). 


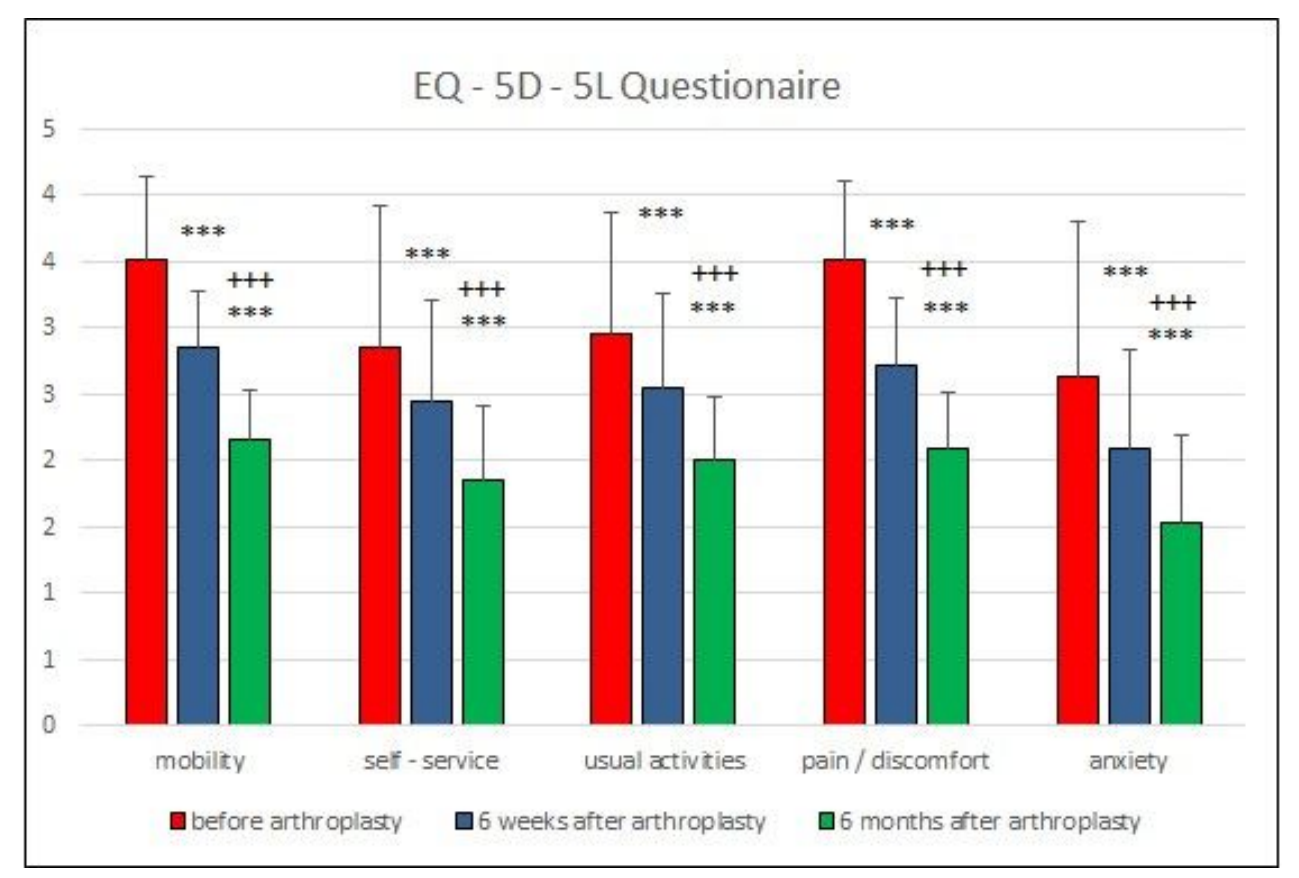

Figure 3

Assessment of quality of life according to EQ-5D-5L questionnaire before, 6 weeks and 6 months after hip arthroplasty. ${ }^{* \star} p<0.001$ compared to the value before the surgery, $+++p<0.001$ compared to the value after the surgery.

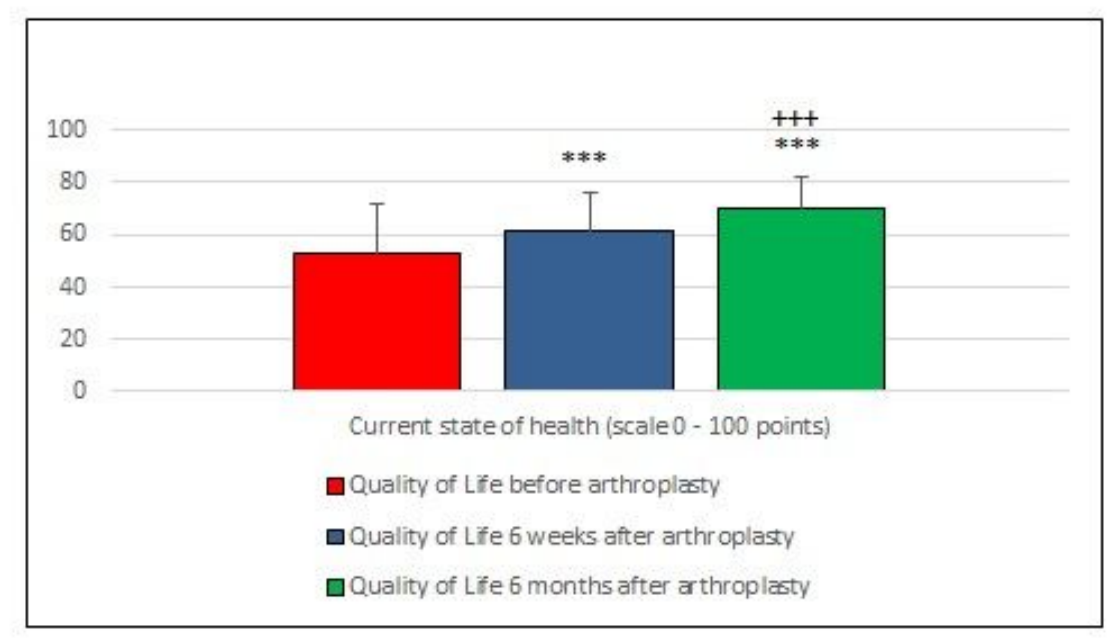

\section{Figure 4}

Subjective health status before, 6 weeks and 6 months after hip arthroplasty. $* * p<0.001$ comparedto the value before surgery, $+++p<0.001$ comparedto the value after surgery. 


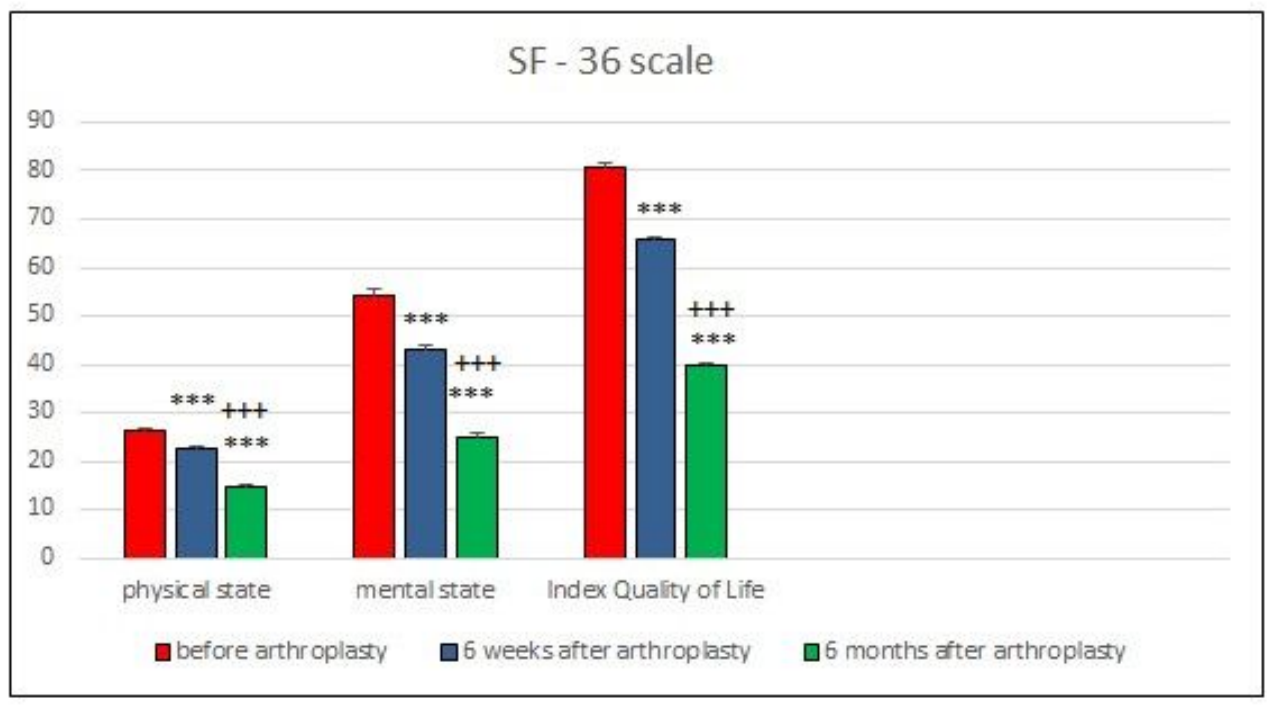

\section{Figure 5}

Assessment of quality of life according to SF-36 questionnaire before, 6 weeks and 6 months after total hip arthroplasty. ${ }^{* \star} p<0.001$ compared to the value before surgery, $+++p<0.001$ comparedto the value after surgery. 